\title{
AVO analysis of thin coalbed with dirt band
}

\author{
Yongxu Lu \\ State Key Laboratory of Coal Resources and Safe Mining, \\ China University of Mining and Technology(Beijing) \\ Beijing, China \\ samhomes@163.com
}

\author{
Suping Peng, Wenfeng Du, Peng Lin, Le Xu \\ State Key Laboratory of Coal Resources and Safe Mining, \\ China University of Mining and Technology(Beijing) \\ Beijing, China \\ samhomes@163.com
}

\begin{abstract}
Zoeppritz equations can only describe the reflection and transmission of plane waves incident on a single interface. It cannot deal with the thin bed case. The presence of dirt band in thin coalbed makes the problem even more complicated. We present an AVO (amplitude variation with offset) analysis of thin coalbed with dirt band. We derive equations to calculate the exact solution of reflection and transmission coefficients. Two models are built, one contains a single thin coalbed and the other one insert a dirt band into the coalbed. Both P-P and P-S reflection coefficients are analyzed with varying incidence angle, dominant frequency of plane wave, thickness and position of dirt band. We conclude: (1) dirt band has great effect on the reflection coefficients; (2) the dominant frequency and thickness of dirt band have greater effect on the reflection coefficients; (3) the position of dirt band may be difficult to be obtained using AVO response because it has little effect on the reflection coefficients.
\end{abstract}

Keywords—AVO; thin coalbed; dirt band;

\section{INTRODUCTION}

Amplitude variation with offset (AVO) technology can be used to detect coalbed methane ${ }^{[3,4]}$. Traditional AVO methods are based on the Zoeppritz equation, which are derived assuming a plane wave incident on a single interface.

However, in many cased in China, coalbeds are thin beds according to the wavelength of seismic waves. The top and bottom reflections of thin coalbeds are interfering, and the results no longer obey the Zoeppritz equation. Widess (1973) studied the thin bed AVO response at normal incidence ${ }^{(5]}$. Liu and Schmitt (2003) gave the analytical solution in acoustic regime $^{[1]}$. Pan and Innanen (2013) extended the thin-bed AVO analysis to elastic media ${ }^{[2]}$. However, these methods can only be applied when a single thin coalbed exists. For some coal mines in China, there are dirt bands inside the thin coalbeds, which makes the problem a thin-interbed problem. The dirt bands are often harder than the coal seam around and their existence can have great influence on the reflection coefficients.

In this research, we derive the equation to calculate the exact solution of reflection and transmission of plane wave incident on a thin-coalbed with dirt band. The algorithm is based on the propagator algorithm proposed by Carcione (2001) and we modified it to fit our problem. We inves $\neg$ tigate the influence of incidence angle, dominant frequency of plane wave, thickness and position of dirt band on the P-P and P-S reflection coefficients and give some conclusions.

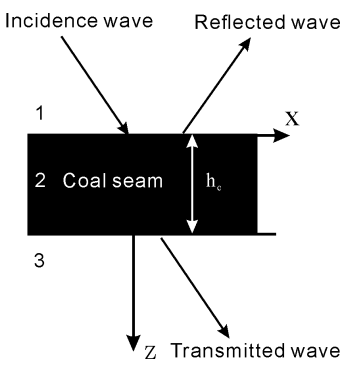

(a)

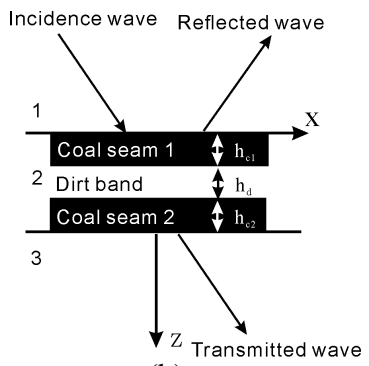

(b)
Fig. 1. Thin coalbed model without dirt band (a) and with dirt band (b).

\section{METHOD}

The geometrical configuration we use is showed in Fig.1, which is a three-layer model. The layer 2 can be thin coalbed only (Fig. 1a) or with dirt band inside (Fig. 1b).

First we consider the single thin coalbed case (Fig.1a). Inside the thin coalbed, there are upgoing and downgoing waves. The particle displacement-stress vector $t(\mathrm{z})$ and displacements of P- and S- waves are connected by a coefficient matrix $\mathrm{T}(\mathrm{z})$ :

$$
\mathbf{t}(z)=\left(\begin{array}{c}
u_{x} \\
u_{z} \\
\sigma_{z z} \\
\sigma_{x z}
\end{array}\right)=\mathbf{T}(z)\left(\begin{array}{c}
U_{P}^{-} \\
U_{S}^{-} \\
U_{P}^{+} \\
U_{S}^{+}
\end{array}\right),
$$

where

$$
\begin{aligned}
\mathbf{T}(z) & =\left(\begin{array}{cccc}
\alpha_{P}^{-} & \alpha_{S}^{-} & \alpha_{P}^{+} & \alpha_{S}^{+} \\
\beta_{P}^{-} & \beta_{S}^{-} & \beta_{P}^{+} & \beta_{S}^{+} \\
D_{P}^{-} & D_{S}^{-} & D_{P}^{+} & D_{S}^{+} \\
E_{P}^{-} & E_{S}^{-} & E_{P}^{+} & E_{S}^{+}
\end{array}\right) \\
& \times\left(\begin{array}{cccc}
e^{-\mathrm{i} \omega S_{z P}^{-} z} & 0 & 0 & 0 \\
0 & e^{-\mathrm{i} \omega S_{z S}^{-} z} & 0 & 0 \\
0 & 0 & e^{-\mathrm{i} \omega S_{z P}^{+} z} & 0 \\
0 & 0 & 0 & e^{-\mathrm{i} \omega S_{z S^{\prime} z}^{+}}
\end{array}\right)
\end{aligned}
$$




$$
\begin{gathered}
D=-\lambda S_{x} \alpha-(\lambda+2 \mu) S_{z} \beta, \\
E=-\mu\left(S_{z} \alpha+S_{x} \beta\right),
\end{gathered}
$$

where $\omega$ is the circular frequency, $U$ is the wave amplitudes, vector is polarization of plane wave, Sx and Sz are horizontal and vertical slowness, superscript - and + correspond to the upgoing-waves and the downgoing-waves, $\lambda$ and $\mu$ are Lamé parameters. We assume the interface between layer 1 and layer 2 are at $\mathrm{z}=0$ for simplicity. Then the particle displacement-stress vector at $\mathrm{z}=0$ and $\mathrm{z}=\mathrm{h}_{\mathrm{c}}$ are connected as

$$
\mathbf{t}(0)=\mathbf{B t}\left(h_{c}\right),
$$

where $\mathrm{B}=\mathrm{T}(0) \mathrm{T}-1(\mathrm{hc})$.

At boundary of layer 1 and layer 2, the particle displacement-stress vector $t(0)$ is continuous. Then the $t(0)$ can be expressed as:

$$
\mathbf{t}(0)=\mathbf{A}_{1} \mathbf{r}+\mathbf{i}_{P}
$$

where

$$
\begin{aligned}
\mathbf{r} & =\left[R_{P P}, R_{P S}, T_{P P}, T_{P S}\right]^{T}, \\
\mathbf{i}_{P}= & {\left[\alpha_{P}^{(1)-}, \beta_{P}^{(1)-}, D_{P}^{(1)-}, E_{P}^{(1)-}\right]^{T}, } \\
\mathbf{A}_{\mathbf{1}} & =\left(\begin{array}{llll}
\alpha_{P}^{(1)+} & \alpha_{S}^{(1)+} & 0 & 0 \\
\beta_{P}^{(1)+} & \beta_{S}^{(1)+} & 0 & 0 \\
D_{P}^{(1)+} & D_{S}^{(1)+} & 0 & 0 \\
E_{P}^{(1)+} & E_{S}^{(1)+} & 0 & 0
\end{array}\right),
\end{aligned}
$$

and the superscript (1) represents for layer $1, R$ and $T$ are reflection and transmission coefficients, respectively. $\mathbf{i}_{\mathrm{P}}$ is for incident $\mathrm{P}$ wave and $\mathbf{A}_{1}$ is for reflected $\mathrm{P}$ - and $\mathrm{S}$ - waves. Similarly, the $\mathbf{t}\left(\mathrm{h}_{\mathrm{c}}\right)$ can be expressed according to layer 3:

$$
\mathbf{t}\left(h_{c}\right)=\mathbf{A}_{3} \mathbf{r},
$$

where

$$
\begin{aligned}
\mathbf{A}_{3} & =\left(\begin{array}{llll}
0 & 0 & \alpha_{P}^{(3)-} & \alpha_{S}^{(3)-} \\
0 & 0 & \beta_{P}^{(3)-} & \beta_{S}^{(3)-} \\
0 & 0 & D_{P}^{(3)-} & D_{S}^{(3)-} \\
0 & 0 & E_{P}^{(3)-} & E_{S}^{(3)-}
\end{array}\right) \\
& \times\left(\begin{array}{cccc}
0 & 0 & 0 & 0 \\
0 & 0 & 0 & 0 \\
0 & 0 & e^{-\mathrm{i} \omega S_{2 P}^{(3)} h_{c}} & 0 \\
0 & 0 & 0 & e^{-\mathrm{i} \omega S_{2 S}^{(3)} h_{c}}
\end{array}\right),
\end{aligned}
$$

and A3 is for transmitted P- and S- waves. Substitute equations 5-10 into equation 4 , the refection and transmission coefficients vector $\mathrm{r}$ can be obtained as:

$$
\mathbf{r}=-\left(\mathbf{A}_{\mathbf{1}}-\mathbf{B} \mathbf{A}_{3}\right)^{-1} \mathbf{i}_{P} .
$$

Eq.(11) is for a single thin-bed case. If we consider the existence of dirt band, then layer 2 is a thin-interbed and the equation 11 should be modified to:

$$
\mathbf{r}=-\left(\mathbf{A}_{\mathbf{1}}-\left(\prod_{k=1}^{N} \mathbf{B}_{k}\right) \mathbf{A}_{3}\right)^{-1} \mathbf{i}_{P},
$$

where $\mathrm{N}$ is the layer number of the thin-interbed, and $\mathrm{Bk}=\mathrm{T}(0) \mathrm{T}-1(\mathrm{hk}), \mathrm{k}=1,2, \cdots, \mathrm{N}$.

\section{EXAMPLES}

\section{A. Comparison of AVO Responses for Thin Coalbed with and without Dirt Band}

Eq. (11) and (12) can be used to analyze and compare the AVO responses of thin coalbed with and without dirt band. The dominant frequency is fixed to $20 \mathrm{HZ}$ and the incidence angle varies from 0 degree to 50 degree. The model can be seen in figure 1 and the corresponding parameters are listed in table 1. The dirt band is embedded in the middle of the coal seam. Fig2a and 2b show the real reflection coefficients of P-P and P$\mathrm{S}$ waves, respectively. It can be seen that the real reflection coefficients of both cases have similar trends. The real Rpp is increasing with incidence angle. For Rps, the value firstly increasing than decreasing with incidence angle. However, the real Rpp of thin coalbed with dirt band are larger than that without dirt band, and for real Rps, it is the other way around. For real Rpp, the difference decreases with the incidence angle increasing. The maximum difference is about 0.1 when the incidence P-wave is normal to the interface. For real Rps, the difference is 0 for normal incidence because no convertedwave are generated, and become larger with the incidence angle increasing.

TABLE I. MODEL PARAMETERS.

\begin{tabular}{c|c|c|c|c|c}
\hline \multicolumn{2}{c|}{ Layer } & $\begin{array}{c}V_{P} \\
(\mathrm{~m} / \mathrm{s})\end{array}$ & $\begin{array}{c}V_{S} \\
(\mathrm{~m} / \mathrm{s})\end{array}$ & $\begin{array}{c}\rho \\
\left(\mathrm{kg} / \mathrm{m}^{3}\right)\end{array}$ & $\begin{array}{c}\text { Thickness } \\
(\mathrm{m})\end{array}$ \\
\hline \multirow{2}{*}{2} & 3000 & 1700 & 2200 & $\infty$ \\
\hline \multirow{2}{*}{2} & $\begin{array}{c}\text { Coal } \\
\text { seam }\end{array}$ & 1400 & 800 & 1200 & $\begin{array}{c}8 \\
\text { (including } \\
\text { dirt band } \\
\text { if exist })\end{array}$ \\
\cline { 2 - 6 } & $\begin{array}{c}\text { Dirt } \\
\text { band }\end{array}$ & 2000 & 1200 & 1800 & 2 \\
\hline \multicolumn{2}{c|}{3} & 3000 & 1700 & 2200 & $\infty$ \\
\hline
\end{tabular}

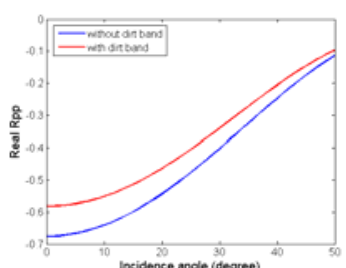

(a)

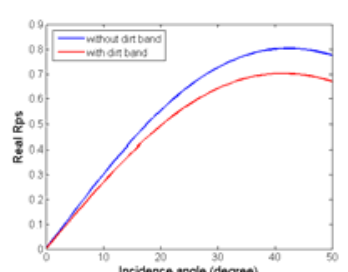

(b)
Fig.2. AVO responses of thin coalbed with and without dirt band. (a) Real Pwave reflection coefficients; (b) Real S-wave reflection coefficients. The blue and red lines are thin coalbed without and with dirt band, respectively. 


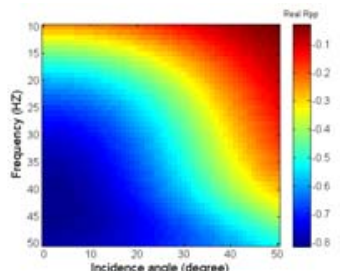

(a)

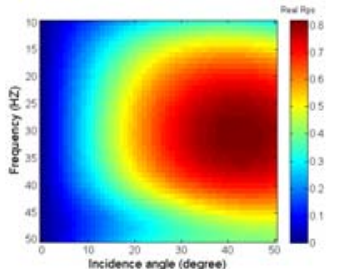

(b)
Fig.3. AVO responses of P-P (a) and P-S (b) waves with changing dominant frequency and incidence angle.

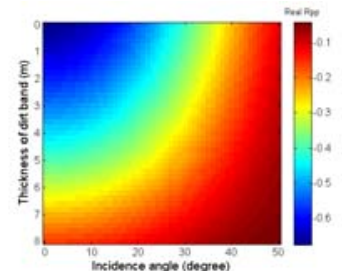

(a)

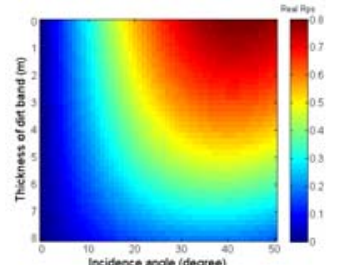

(B)
Fig. 4. AVO responses of P-P (a) and P-S (b) waves with changing thickness of dirt band and incidence angle.

\section{B. The Effects of Frequency of Dirt Band on the Reflection Coefficients}

The P-P and P-S reflection coefficients are functions of dominant frequency. We use same dirt band model in Fig 2 with frequency changing from $10 \mathrm{HZ}$ to $50 \mathrm{HZ}$. The results are shown in Fig. 3. It can be seen that at fixed frequency, the P-P and P-S reflection coefficients become larger with increasing incident angle. And at fixed incident angle, the P-P reflection coefficients become smaller as the dominant frequency increasing. Rps, however, become ups and downs.

\section{The Effects of Thickness of Dirt Band On the Reflection Coefficients}

The thickness of the dirt band is another factor that have influence on the reflection coefficients. We use the same model in Fig.3, with the thickness of the dirt band varying from $0 \mathrm{~m}$ to $8 \mathrm{~m}$. In fact, $0 \mathrm{~m}$ thick means coalbed without dirt band, and $8 \mathrm{~m}$ thick is the case that whole coalbed is replaced by the dirt band. Results are shown in Fig.4. As one can see that thickness of the dirt band has great influence on reflection coefficients. The P-P reflection coefficients are increasing with the dirt band thickening when incidence angle is fixed. Rps, however, show a contrary tendency.

\section{TheEffects ofPosition of Dirt Band on the ReflectionCoefficients}

In the above examples, dirt band is placed in the middle of the coalbed, which are not always the case in the real world. The variation of dirt band position will have influence on the interfering of the reflected waves from top and bottom of the thic coalbed, and finally affect the reflection coefficients. To evaluate the effects of position on reflection coefficients, we use same model in Fig. 3. We chang the thickness of coal seam 1 from $0 \mathrm{~m}$ to $6 \mathrm{~m}$ and the thickness of dirt band and layer 2 remain unchanged. Fig. 5 is the P-P and P-S reflection coefficients with varying thickness of coal seam 1 and incidence angle. Both P-P and P-S reflection coefficients are

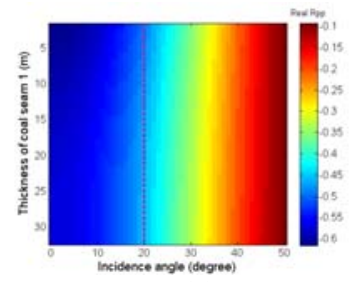

(a)

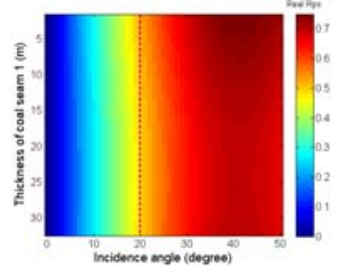

(b)
Fig.5. AVO responses with varying thickness of coal seam 1 and incidence angle. (a) Real Rpp. (b) Real Rps.

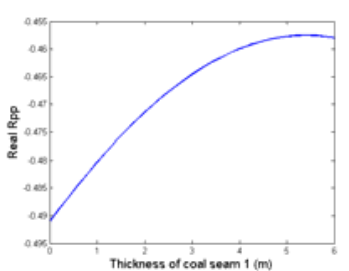

(a)

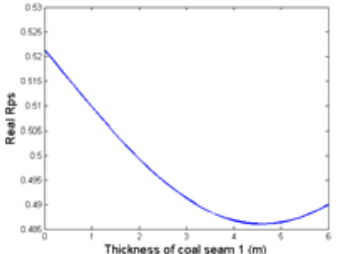

(b)
Fig.6. Rpp (a) and Rps (b) with varying position fo dirt band. Incidence angle are fixed to 20 degree.

varying with difference position of dirt band when incidence angle is fixed. However, the variation seems quite small. To see more detail, we fix the incidence angle to 20 degree (purple-dashed line in Fig.5) and get two curves of the relationship between reflection coefficients and position of dirt band. They are shown in Fig.6. One can see that the Rpp is increasing with thickness of coal seam 1 when the thickness is less than about $5 \mathrm{~m}$. When the thickness keep increasing, the Rpp decrease. The Rps show the opposite pattern to Rpp. However, the differences between the maximum and minimum values of reflection coefficients are relatively small (about 0.037 for Rpp and 0.04 for Rps). This indicates that estimate the position of dirt band using AVO responses is difficult.

\section{CONCLUSION}

Traditional AVO techniques based on the Zoeppritz equation is not suitable for the thin coalbed cases. The existence of dirt band makes the problem more complicated. The AVO response of P-P wave has larger difference between coalbed with and without dirt band when incidence angle is small. For Rps, the difference is zero for normal incidence and increases with incidence angle. The dominant frequency of plane wave and thickness of dirt band have great impact on the reflection coefficients. The effects of position of dirt band on reflection coefficients, however, are relatively small.

\section{ACKNOWLEDGMENT}

We thank the editors and anonymous reviewers' help to improve our paper.

\section{REFERENCES}

[1] Y. Liu, D.R. Schmitt, "Amplitude and AVO responses of a single thin bed,” Geophysics, vol. 68, pp. 1161-1168, August 2003. 
[2] W. Pan and A.K. Innanen, "AVO/AVF analysis of thin-bed in elastic media,” 83th Annual International Meeting, SEG, Expanded Abstracts, America, 2013, pp. 373-377.

[3] S.P. Peng, Y.F. Gao, R.Z. Yang, H.J. Chen and X.P. Chen, "Theory and application of AVO for detection of coalbed methane: A case from the Huainan coalfield," Chinese Journal of Geophysics, vol. 48, pp. 14751485, November 2005 (In Chinese).
[4] A.C.B. Ramos and T.L. Davis, "3-D AVO analysis and modeling applied to fracture detection in coalbed methane reservoirs,” Geophysics, vol. 62, pp. 1683-1695, November 1997.

[5] M.B. Widess, "How thin is a thin bed?" Geophysics, vol. 38, pp. 11761180, December 1973. 